\title{
On the Superiority of Translators Over Machines*
}

\author{
REN Rui, ZHANG Lele \\ Northeastern University, Shenyang, China
}

\begin{abstract}
Translation determines the effect of the popularization of Chinese culture in other languages and cultures. And the cultivation of translators is an essential part of the building of China's cultural soft power. In recent years, with the development of technology, machine translation has become a hot topic in translation field and developed in a strong momentum. It has aroused widespread discussion in translation field on whether the machine translation can replace human translators. This paper argues that machine translation cannot replace human translators. There are three subtleties: human-oriented subtleties, linguistic-cultural subtleties, social-cultural subtleties, which play an important role in the translation and interpretation.
\end{abstract}

Keywords: machine translation, human translation, superiority

\section{Introduction}

With the coming of globalization, people have more and more frequent communication and cooperation in trade and commerce, culture, sport and tourism and the other areas, and more and more language communicating problems are occurring, which results in the huge demand for machine translation. Machine translation refers to a science of transferring the source language into the target language by computer. It involves five parts, the input of source language, the analysis of source language, the transfer from source language to target language, the form of target language, and the output of target language. The concept of machine translation was initially proposed by a France scientist in 1930s.

With the fast development and broad application of machine translation, many translation practitioners, even the students who major in translation or interpretation, are worrying about that machine translation may replace human beings. Can machine translation take the place of human translators or interpreters? This article argues that the machine translation cannot replace human. Though the machine translation has many advantages, such as fast and convenience, it doesn't have the flexibility, creativity, and analytical ability that human beings have and are necessary for translating.

\section{Human-Oriented Subtleties}

Human-oriented subtleties, as a fundamental question, include the following two subtleties: linguistic-cultural and social-cultural. In other words, the linguistic-cultural subtleties and social-cultural

\footnotetext{
* Acknowledgements: This paper is supported by the Youth Project of China's Philosophy and Social Science Fund "The Study on Operational Norms of Chunks Based on Chinese-English Interpreting Corpus (No. 17CYY052)”, the Fundamental Research Funds for the Central Universities “An Exploration into Innovation of Translation Competence Driven by Translation Technology (N171501003)”, and 2017-2018 Projects of Reforms on Experiments and Practice of Postgraduate Programs at Northeastern University.

REN Rui, associate professor, M.A., English Department, Northeastern University, Shenyang, China.

ZHANG Le-le, master candidate, English Department, Northeastern University, Shenyang, China.
} 
subtleties can be subscribed to human-oriented subtleties, which can be elaborated from three aspects, the tones, body language, and volume.

\section{Tones}

Tones play a critical role in understanding and transferring information. Here, I'd like to take news commentary as an example to elaborate in detail.

World food prices are pushing higher-the United Nations overall food index shows a $28.3 \%$ annual increase, with cereals up $44.1 \%$ - sparking concerns that a new food crisis may be emerging, just three years after the last one.

In this example, the words "emerging" and "just" show the author's sense of urgency, anxiety, and concerning. Therefore, when we translate this kind of sentence, we need to demonstrate this kind of feeling in the target language. The machine translation will not notice that point.

\section{Body Language}

In some cultures, especially the high-context culture, non-verbal communication may convey more information than the verbal communication. Therefore, the body language is a necessary part in communication. I'd like to take the gestures and facial expressions as examples.

Shrug, based on different contexts, stands for different meaning, such as unhappy, desperate, surprised, puzzled, smirking, contemptuous, not concerned, and so on. During the process of interpreting, if the speaker does the action of "shrug", the interpreter needs to decide the appropriate meaning of it depends on the context, and then the interpreter should choose appropriate expression or meaning of target language to transfer more information as he/she can. On the contrary, the machine can't do the same thing, not even to transfer the connotation meaning of speaker.

Another example is about facial expression and we all know that smart has the similar meaning with clever, intelligent, remarkable, sophisticated, and so on. If the speaker says: "He is a smart guy", the direct translation is good, but if the speaker says "He is smart guy" while widening his eye and rising his tone, the same sentence has different meaning. The smart here may has an ironic meaning, which may represent the similar meaning as sophisticated or complex.

\section{Volume}

When we have the simultaneous interpretation and consecutive interpretation classes, the professor always tells us that the stable speed, moderate volume, and natural tone are necessary for interpreters. The volume not only can convey the information, but also can make the focal points standout, which will let the listeners or audiences catch the major structure to easily understand the information. In terms of volume, the human interpreters can catch the highlights of a speech; however, the machine cannot do the same thing as human beings.

\section{Linguistic-Cultural Subtleties}

In terms of linguistic-cultural subtleties, cultural subtleties, including spoken language, idioms and slangs, and translation strategies can be subscribed to linguistic-cultural subtleties.

\section{Cultural Subtleties}

As far as the spoken language is concerned, I'd like to give some examples about the speech of Li Keqiang. The machine translated “一家母子二人” into “a mother and two/a mother of two people” without concerning 
the context and the human's translation is “one, there is a mother and son”. The machine translated “住在四面 透风的破瓦房里” into “living in a tiled house surrounded by wind” while the correct translation should be "living in a very shabby place where wind can even be felt when you are in the home". Another example is that the machine translated “好不容易出了一个大学生” into “It’s hard to leave a college student” while the correct translation should be "The other home has cultivated a college student". From all the examples, we can see clearly that all the later versions are better than the former one, which can be understood easily and conveyed appropriately the real meaning of this speech.

Another example is Chinese four-character idioms. For example, the machine translated “起早贪黑” into "Early and late/early and dusk", which cannot be understood at all and the correct version should be "work from dawn to dusk” to convey the sense of laborious. The machine translated “冊庸讳言” as “Needless to say”, which doesn't convey the meaning of saying something directly. If the speaker uses the Chinese four-character idioms consecutively, for example, “只要我们铸而不舍, 假以时日, 必有成效”, the machine translated this sentence into "As long as we are perseverant, with time, we must be effective" while the correct translation should be "As long as we have persistent efforts over time, we can make it a success".

The last instance is about the slangs, for example, “笑掉大牙” should be translated into "Laugh one’s head off” rather than "Laugh big teeth”; “落汤鸡” should be translated into "drawn rat” rather than "drenched chicken" because the there have many rats in the western countries' drainpipes. Besides, "turn the table" means "turn the situation around" not "overturn the real table". Human translators can identify the tiny difference while the machine cannot transfer the real meaning.

\section{Translation Strategies}

No matter it is translation or the interpretation, in order to transfer the real meaning of speaker, appropriate translation strategies should be used. In terms of translation, we need to do the supply or delete according to the context, especially when the speakers don't have certain logic. Jack Ma is a special example of not having logic. The following is a speech fragment of Jack Ma in Stanford University.

It's my great honor and, I was invited about months ago. I did not expect, people said this is very sensitive time for me to talk, because about yahoo, about Alibaba about this, that, so I promise is promise, I come. Today any question you have, I will answer any question you have. All right, so I've been here for fifteen day and I planned to spend one year here. Nobody knows, my company did not know that, I am gonna spend one year here. Cause people asked why you are here. Are you here for getting yahoo? "No".

When we translate the speech of such, we need to transfer the information as much as we can. The interpreters should extract the major information, comb the content, and find the logic.

\section{Social-Cultural Subtleties}

The social label and social position of a people can determine his/her language style, which will bring power distance. If we don't concern the feeling of the audience, the cultural concern and misunderstanding will occur. This chapter will talk about the social-cultural subtleties from political concerns, conflicts, and jokes, three aspects. In terms of these three aspects, the interpreters exist as the cultural mediation.

\section{Political Concerns}

During the process of translation and interpretation, the interpreters need to pay attention to the politically sensitive problems, especially for the diplomatic interpretation. When the translation or interpretation concerns 
the politically sensitive problems, the translators need to deal with the political concerns. Sometimes the interpretation will be about the political stands. For example, in interpreting, sometimes the leader will have a slip of the tongue. At this time, the translator has a function of correcting errors because automatic error correction can help correct the logical and simplistic places. When Nixon visited China, the central leading comrade was not used to saying Mr. Nixon. When he said, he said "Comrade Nixon". At this time, the human interpreters can correct it appropriately by interpreting "comrade" to "Mr." But the machine cannot translate like this.

\section{Conflicts}

In the process of interpretation or translation, there always have some kinds of conflicts, especially for the negotiation. When the conflicts occur, the interpreters can take appropriate measures, such as adopting euphemism, fuzzy language, or deleting methods, and so on. Human translators or interpreters can mediate the negative factors that can result in the failure of communication during the process of interpreting. For example, the communicators of both sides may have some insult or impolite words, at this time, the human translators can mediate it appropriately in order to achieve successful communication.

\section{Jokes}

Translation or interpretation is involving the communication between two different cultures. Therefore, the cultural conflicts certainly exist here. For example, when the speaker uses jokes to break the ice of communication, but the listeners of the target language culture may not understand it very well due to the different cultural backgrounds. The machine translation can transfer the meaning of this joke, but the effect and the original purpose of speaker cannot be achieved because the jokes are not understood by the audience. I've heard such a story, a speaker of the United States come to chine to deliver a speech; at the beginning, the speaker told a joke but the translator cannot understand it very well, so the interpreter told the audience that the speaker told a joke and asked them to laugh, and the speaker was very happy.

\section{Conclusion}

The process of human translation is a comprehensive process in which the human translator integrates understanding, analysis, selection, and re-creation into a unit. It reflects the process of brain activities. Admittedly, the machine translation has many advantages, such as fast and convenience. However, the machine does not have the creativity, understanding and analytical ability and flexibility that the human beings have, because of the factors mentioned above, the human-oriented subtleties, linguistic-cultural subtleties, and social-cultural subtleties. In other words, machine cannot replace human because machine translation can only convey messages in a broad sense, but it cannot convey the deeper cultural connotations behind or cultural differences, or different cultural contexts; cannot convey tones, expressions, body languages, which are of vital importance in cross-cultural communication; cannot adopt appropriate skills and strategies in the process of interpreting and translation, such as adding, supplementing, or omitting, in order to achieve the goal of smooth cross-cultural communication. However, at current, machine translation can be used as a tool to assist human to do translation or interpretation.

\section{References}

Hutchins, W. J., \& Sommers, H. I. (1992). An introduction to machine translation [M]. San Diego: Academic Press Inc. 
Barkhudarov. (1985). Language and Translation [M]. translated by Caiyi. Beijing: China Foreign Translation and Publication Corporation.

Li, Y. J. (2014). Study on the Present Situation and Prospect of Machine Translation and Translation Technology [J]. Chinese Science \& Technology Translators Journal.

Ye, Z. N. (2001). Advanced English-Chinese Translation [M]. Beijing: Tsinghua University Press.

Zhao, M. (2007). On the Translation Strategy and Method of Chinese English [J]. China University of Mining and Technology (Social Sciences Edition).

Zhang, Z. (2006). The Study of Computer Translation [M]. Beijing: Tsinghua University Press. 